\title{
Expectations for Direct Photon Physics from Fermilab Experiment E705
}

D. E. Wagoner ${ }^{8)}$, M. Arenton ${ }^{11}$, T. Y. Chen ${ }^{1}$, S. Conetti ${ }^{8)}$, B. Cox ${ }^{4}$, S. Delchamps ${ }^{4}$,

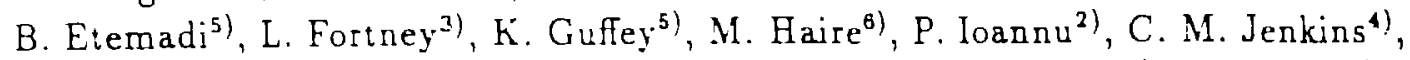
D. J. Judd ${ }^{\text {() }}$, C. Kourkoumelis ${ }^{2}$, I. Koutentakis ${ }^{2}$, J. Kuzminski ${ }^{6}$, K. W. Lail ${ }^{1}$, A. Mianousakis-Katsikakis ${ }^{2}$, H. Mao ${ }^{9}$, A. Marchionni ${ }^{6)}$, P. O. Mazur ${ }^{4}$, C. T. Murphy ${ }^{4}$, T. Pramantiotis ${ }^{2}$, R. Rameika ${ }^{4}$, L. K. Resvanis ${ }^{3)}$, M. Rosati ${ }^{6}$, J. Rosen ${ }^{7}$, C. H. Shen ${ }^{9}$, Q. Shen ${ }^{3)}$, A. Simard ${ }^{6)}$, R. Smith ${ }^{4}$, L. Spiegel ${ }^{7)}$, D. Stairs ${ }^{6)}$, R. Tesarek ${ }^{3)}$, W. Tucker ${ }^{5}$, T. Turkington ${ }^{3)}$, F. Turkot ${ }^{4)}$, L. Turnbull( $\left.{ }^{6}\right)$, S. Tzamarias ${ }^{7)}$, M. Vassiliou ${ }^{2}$, G. Voulgaris ${ }^{2)}$,

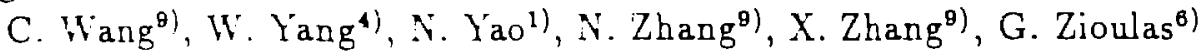

\author{
1) University of Arizona, Tucson, Arizonu \\ 2) University of Athens, Athens, Greece \\ 3) Duke University, Durham, North Carolina : 1: ... \\ 4) Fermilab, Batavia, Mlinois \\ 5)Florida A\&M University, Tallahassee, Florida \\ a) McGill University, Montreal, Quebec, Canada \\ 7) Northwestern University, Evanston, Illinois \\ 8) Prairie View A\&M University, Prairie View, Texas \\ ${ }^{9}$ Shandong University, Jinan, Shandong, People's Republic of China
}

November, 1987

\begin{abstract}
The E705 scintillation glass/lead glass electromagnetic calorimeter is described. The trigger used for recording high transverse momentum direct photon signals from $300 \mathrm{GeV} / \mathrm{c}$ $\pi^{-}, \pi^{+}, \bar{p}, p$ interactions in a $\mathrm{Li}^{\top}$ target is explained. Preliminary results on the response of this direct photon trigger and electromagnetic calorimeter and expected event sensitivities are prestented.
\end{abstract}

To be published in Praceedings of the Advanced Research Workshon on QCD Hard Hadronic Processes, St. Croix, Virgin Islands, October 8-13, 1987. 


\section{Introduction}

Experiment ET051', at Fermilab is a fixed target experiment to st:1dy charmonium and direct photon production with $300 \mathrm{GeV}^{\circ} \mathrm{c}$ beams of $\pi^{-}, \pi^{+}, \bar{p}$, and $p$ on a $\mathrm{Li}^{7}$ target. This experiment is currently in the middle of its data taking run so any results reported here are strictly preliminary. This paper will describe the direct photon goals of E7us and the electromagnetic calorimeter and direct photon trigger used in the experiment. The charmonium part of the experiment is described in these proceedings in Rrf. [2].

Direct Photon Physics Goals of ET05

Production of high transverse momentum direct photons provides a clean method to probe the parton nature of hadrons and to perform tests of the theory of QCD. Refs. [3] and 4] are excellent reviews on direct photon production in QCD and a theoretical review of this topic is also presented in these proceedings in Ref. [5]. A goal of E705 is to study the contributions of the annihilation and Compton processes to diract photon production at $\sqrt{s}=23.7 \mathrm{GeV} / \mathrm{c}$ by performing comparisons of $\pi^{-}$vs $\pi^{+}$and $\bar{p}$ vs $p$ direct photon production for $p_{T}>4 \mathrm{GeV} / \mathrm{c}$. E705 also plans to study leading particle distributions from the recoil quark or gluon fragmentation and measure the nucleon and pion structure functions in the accessible kinematic range. The study of high mass diphoton production is also another goal of E705.

In particular by studying the difference of direct photon cross sections between the beam types, i.e., $\sigma_{\gamma}\left(\pi^{-} \mathrm{Li}\right)-\sigma_{\gamma}\left(\pi^{+} \mathrm{Li}\right)$ and $\sigma_{\gamma}(\bar{p} \mathrm{Li})-\sigma_{\gamma}(p \mathrm{Li})$, one can cleanly isolate the quarkantiquark annihilation term. In this way the annihilation and Compton contributions can be separated and valence quark and gluon structure functions for the pion and nucleon can be extracted. Also by studying the difference distributions for the characteristics of the recoil leading particles one can attempt to determine properties of gluon and quark fragmentation.

\section{E705 Exper ascalal Apparatus}

E705 is a large aperture open geometry fixed target experiment. Fig. 1 shows the layout of the experiment. The beam used is a $300 \mathrm{GeV} / \mathrm{c} 98.5 \% \pi^{-} / 1.5 \% \overline{2}$ or $40 \% \pi^{+} / 60 \% p$ beam. The beam particle types are tagged with two gas threshold Cherenkov counters and the bearn trajectory is measured at three beam PWC stations. The target is $33 \mathrm{~cm}$ of $\mathrm{Li}^{7}$.

Between the target and the analysis magnet are three PWC chamber sets and three drift chamber sets. These chamber sets are deadened in the beam region to allow the noninteracted beam to pass through without producing hits. To detect particles in the beam region there are three PWC chamber sets with finely spaced wires which are sensitive only in the beam region. The aperture covered by the combination of these nine chamber sets 
matches the outer aperture of the analysis magnet which is $=142 \mathrm{mrad} x=81 \mathrm{mrad}$. The analysis magnet has an equiralen $p_{T}$ kick of $0.766 \mathrm{GeV} / \mathrm{c}$. Following the analysis magnet are three large drift chamber stations with deadened beam regions. These sets of upstream and downstream chambers will permit ET05 to perform tracking on leading particles from the direct photon recoil iragmentation. Some level of $K_{S}^{0}$ reconstruction can also be done.

Following the downstrearu drift chambers are two sets of finely segmented scintillation hodoscope planes one oriented horizontally and the other vertically. Then follows the electromagnetic calorimeter which will be described more fully later. Finally there is the steel muon absorber and tungsten beam dump. The muon absorber kas a stopping power of $6 \mathrm{GeV} / \mathrm{c}$ and contains within it the muon scintillator hodoscopes. There are three vertically oriented planes of muon hodoscope and one horizonially oriented plane. These muon hodoscopes are to define the dimuon trigger used in the charmonium part of E705.

\section{E705 Electromagnetic Calorimeter}

Fig. 2 shows a side view of the E705 electromagnetic calorimeter. It is located $10 \mathrm{~m}$ from the target and is composed of three parts: i) the main array (the downstream part on the right), ii) the active converter/lead-gas converter (the upstream part on the left), and iii) the gas tube hodoscope (which is located in the gap between the active converters and main array). The active converter/lead-gas converter/gas tube hodoscope is used to start the electromagnetic shower and determine its position. They are also used to provide rejection against hadion showers. The main array is used to determine the energy of the electromagnetic siower. These devices are now described mere fully.

Main Array

Fig. 3 shows a froni view of the main array of the E705 electronagnetis calorimeter. The area inside tha dashed lines contain SCG1-C scintillation glass blocks 21 radiation lengths $\left(\mathrm{X}_{0}\right)$ long and the blocks outside are SF5 lead glass $18 \mathrm{X}_{0}$ long. The small SCG1-C blocks in the center are $7.5 \mathrm{~cm} \times 7.5 \mathrm{~cm}$ in transverse size and are read out with RCA $6342 \mathrm{~A}$ photomulaipliers. The large SCG1-C and SF5 blocks are $15 \mathrm{~cm} \times 15 \mathrm{~cm}$ and are read out with EMI $9791 \mathrm{~KB}$ photomultipliers. There is an empty beam hole of size $\pm 15 \mathrm{~cm} \times \pm 7.5 \mathrm{~cm}$.

SCG1-C is a heavy Barium glass containing an inorganic scintillator that is produced by Ohara Optical Glass Manufacturing Co., Ltd. SCG1-C has been tested extensively by ET05:6) and some of its properties compared to SF5 are given in Table 1. The two main advantages of SCG1.C are that it produess over 5 times as much light as SF5 (which implies that its energy resolution should be at least a factor of 2 better than that for SF5) and that it is $\approx 150$ more resistant to radiation darkening than SF5. Fig. 4 shows the energy resolutiun of SCG1-C as measuret in Ref. [6]. The energy resolution for E705 conditions 
Table 1: Properties of SCG1.C scintillation glass and SF5 lead glass '6'.

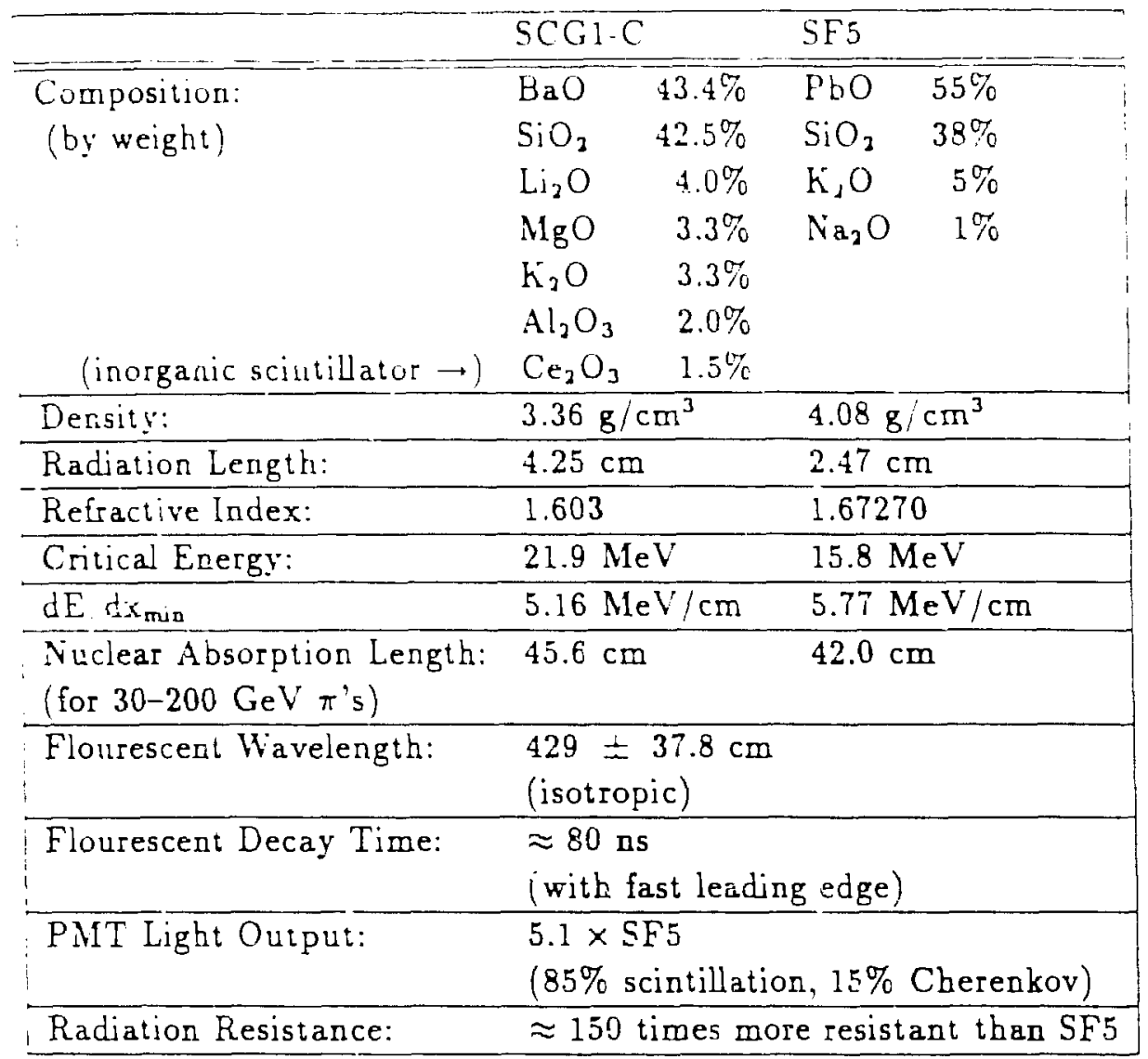

is expected to be: $\sigma_{E} / E \approx 1.2 \%+2.0 \% / \sqrt{E}$ (with $E$ in $\mathrm{GeV}$ ). Fig. 5 shows the increased radiation resistance of SCG1-C compared to that of SF5 as determined in Ref. [6].

All 392 channels of the main array go to a precision ADC (with 15 effective bits) specially built for E705. The signals of all main array channels also go to JeCroy 4290 TDC's to determine the time of the various energy deposits. Each block has mounted on its upstream face an optical fiber coming fiom the central LED pulsing system use i to monitor the gain of each block between electron calibration runs.

All channels of the main array also go the fast analog cluster finder which is used to form the high $p_{T}$ direct photon trigger and diphoton trigger used in E705. The outermost layer of SF 5 blocks and innermost laver of small SCG1-C blocks are not in the trigger fiducial volume and are not used to produce direct photon or diphoion triggers; they are used only to cortribute energy as neighbors to clusters wiihin the trigger fiducial volume. 
Fig. 6 shows a front view of the SCG1-C active converters and the central lead-gas converter (LGC). The LGC is an 8 layer lead/proportional tube sandwich occupying the central $=52.5 \mathrm{~cm} \times=97.5 \mathrm{~cm}$ region of the calorimeter. The LGC is a total of $3.8 \mathrm{X}_{0}$ deep and has a beam hole matching that of the main array. The anode segmentation is $1 \mathrm{~cm}$ with similar segmentation for cathode strip readout in the other dimension. The 8 longitudinal samples are ganged together in both the anode and cathode and the pulse height of each ganged signal is read out using a 12 bit LeCroy 2280 ADC system. This device will enable E705 to improve the position resolution and two photon separation over that of the SCG1-C active converter /gas tube hodoscope system and should not substantially degrade the energy resolution of the main array to the high energy photons expected in the central part of the calorimeter.

Two longitudinal layers of active converter blocks of SCG1-C lie to either side of the central LGC. Each layer of SCG1-C is $1.75 \mathrm{X}_{0}$ deep and there is a pre-converter of $1 \mathrm{X}_{0}$ of steel upstream of the first layer. The transverse size of the active converter is $7.5 \mathrm{~cm} \times$ $97.5 \mathrm{~cm}$ and the blocks are read out with RCA 6342A photomultipliers. The two longitudinal measurements of the shower development in the active converter will be used in icientifying photons and electrons and rejecting hadrons. Each active converter block has attached to it an optical fiber from the E705 central LED pulsing system which is used to monitor the gain. Each channel of the active converter is digitized with the E705 precision ADC system that is also used for the main array; the active converter is not used in the formation of the direct photon or diphoton trigger.

\section{Gas Tube Hodoscope}

The gas tube hodoscope contains two planes of conducting plastic proportional tubes with induced cathode strip readout. It is placed between the active converter and main array and covers the same area as the active converter. The propcrtional tubes have a $0.7 \mathrm{~cm}$ spacing. This device is used to determine the electromagnetic shower position for showers originating in the active converter. The properties and resolution of the gas tube hodoscope have been studied in test beams and are reported in Ref. 7. The anode and cathode signals from the two longitudinal layers are ganged together and digitized with a 12 bit LeCroy 2280 ADC systern. The measured position resolution from Ref. [7] of this device is shown in Fig. 7.

\section{Electromagnetic Calorimeter-General}

The entire E705 electromagnetic calorimeter contains 25 tons of glass and sits inside an enclosed temperature controlled house in order to control temperature dependent gain variations. The temperature is controlled though banks of thermoelectric coolers and can 
be kept stable to $=0.06^{\circ} \mathrm{C}$. The house containing the calorimeter is moveable and can be driven vertically and horizontally under computer control to $=0.5 \mathrm{~mm}$. This enables each main array block to be positioned into a calibration electron beam for determining absolute calorimeter calibration constants. The calibration beam is an electron or positron beam with a tunable energy from 2-100 GeV. Electrons are Cherenkov identified and can be momentum tagged.

For monitoring the gain between calibration runs, each glass block has attached to it an optical fiber coming from a central LED pulseri8!. 92 Bewlett-Packard HLMP-3950 LED's are driven by fast triggered transistor avalanche pulsers and the light from these LED's are mixed into a single light source. This single LED light source then passes through a computer controlled filter wheel and is viewed hy a bundle of quartz opt al fibers which are fanned out and attached to the glass blocks of the calorimeter. The LED light source is iemperature controlled by the same system used for the calorimeter and is monitored by 3 PIN photodiodes attached to 3 of the optical fibers. The light level of the LED pulser is stable to $=1 \%$. With this systern the gain of the calorimeter glass blocks can be accurately tracked between calibration runs.

\section{E705 Direct Photon and Diphoton Trigger}

The high $p T$ ciirect photon trigger in E705 is formed from a specially built system[9]. In general, the photon rrigger forms analog energy sums from adjacent main array blocks to find energy clusters. The peak block and energy sum of each cluster are found in paraliel for all clusters. The cluster energies are converted to iransverse momentum and this $p_{T}$ level can then be triggered on. The operation of this trigger will now be explained in more detail.

The signal from each main array block goes into front end electronics. The front end is effectively a charge integrator with pole-zero correction to compensate for the various input signal shapes coming from the various block and PMT types. In order to correct for pile-up and permit high rate operation, baseline restoration is performed using a using a system of two track and holds to perform a "before-trigger" level subtraction. During read out this "before" level is also digitized with a 3 bit $A D C$.

The baseline corrected signal then goes to the precision ADC. This ADC is an autoranging 12 bit ADC with 15 bit sensitivity. The signal is sampled early and a decision is made whether or not to turn on an $8 \mathrm{X}$ amplifier. In this way a fast 12 bit $\mathrm{ADC}$ can be used which will effectively bave 15 bit sensitulty. Tis permits sensitivity to a large dynamic range in energy deposition of from $6 \mathrm{MeV}$ to $20 \mathrm{GeV}$.

The front end also forms the analog output signal which is used by the cluster finder and high $F_{T}$ trigger. This analog signal is formed by the difference of signals from beiore 
and within a tapped delay line. The high pt photon cluster finder finds clusters of energy deposition by forming analog energy sums with each block and its nearest neighbors. These cilister sums are formed through a custom wire-wrapped backplane corresponding to the E705 main array block layout. If a block's energy is larger than that of all its neighbors and larger than a computer setable minimum threshold (currently $4.2 \mathrm{GeV}$ ), then un energy cluster is defined having the position of the peak block and the energy of the analog cluster sum. This analug energy cluster sum is then converted to an analog $p_{T}$ by attenuating the energy of the cluster by an amount proportional to the value of $\sin \theta$ of the peak ulock, i.e., $p_{T} \approx E_{\text {cluster }} \sin \theta_{\text {peak }}$. This analog $p_{T}$ signal is then available for triggering.

Tu form the high $p_{T}$ photon trigger, the main array is divided into quadrarts and each quadrant is hindled independently for trigger formation. Each quadrant ian produce $p_{T}$ triggers at $\&$ different computer setable $p_{T}$ levels which can each be separately prescaled. The $4 p_{T}$ levels (labeled PT1-PT4) are presently set in E705 to 1.7, 2.5, 3.5, and $4.5 \mathrm{GeV} / \mathrm{c}$ where all PT1 $\left(p_{T}>4.5 \mathrm{GeV} / \mathrm{c}\right)$ triggers are read out and the others are appropriately prescaled. Fig. 8 shows preliminary plots of the raw uncorrected $p_{T}$ sums (for the main array only; for each of the $4 p_{T}$ trigger levels $A$ very good correspondence between the $p_{T}$ levels set and triggered on can be seen. A diphoton trigger is implemented by requiring two Łgh $p_{2}$. clusters in opposite quadrants. The lowest $p_{T}$ trigger level is $1.7 \mathrm{GeV} / \mathrm{c}$, so this requires that the triggered diphoton mass be $\geq 3.4 \mathrm{GeV} / \mathrm{c}^{2}$. Since in the E705 cluster finder the cluster summing is analog and all channels are doie in parallel, the cluster finder trigger is quite fast and is capable of operating at a $2 \mathrm{MHz}$ interaction rate.

A preliminary search for $\pi^{0}$ 's has also been performed. Clusters from the two lower $p_{T}$ triggers (PT1 and PT2) were reconstricted in the region outside of the $\mathrm{LGC}( \pm 52.5 \mathrm{~cm}$ ). Fig. 9 shows a preliminary plot of the invariant mass of all combinations of all these reconstructed clusters. No other cuts were made on the invariani mass combinations. A $\pi^{0}$ peak is clearly evident in this plot at the expected mass.

\section{Direct Photon Event Sensitivity}

E705 has a fast CAMAC/VME-based data acquisition sysiem[10] utilizing smart crate controllers and large buffered dual port memory. The E705 trigger and data acquisition

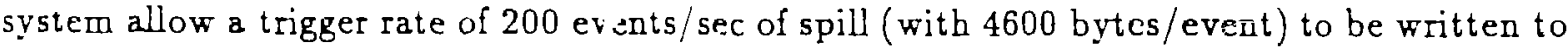
tape. Over half of these triggers are high mass diwuon trigzers[11] used for the charmonium part of the experiment; ihe remainder are deyoted to the sirgle photon and diphoton triggers. To achieve this trigge: rate, E705 is currently rumning at r.n interaction rate of 0.7-1.0 MHz. The duty factor for beam delivery at Fermilab is very favorable with a 23 second long spill once a minute. The E.705 target of $33 \mathrm{~cm}$ of $\mathrm{Li}^{7}$ is $\approx 13 \%$ of an interaction length. With the enhancement due to the $A$ dependence for direct photon processes $\left(\approx A^{1}\right)$ compared to that for total cross section processes $\left(\approx A^{0.72}\right)$, E705 expects to achieve a good sensitivity 
Table 2: Direct photon event sensitivities for various beams in units of events / pb/nucleon ETUS values are based on extrapolated runining through Feb. 15, 1988.

\begin{tabular}{|l|c|c|c|c|c|c|}
\hline Experiment & $\pi^{-}$ & $\bar{p}$ & $\pi^{+}$ & $p$ & Pleb & Target \\
\hline \hline E705 & 9.1 & 0.32 & 5.8 & 6.1 & $\mathbf{3 0 0 \mathrm { GeV } / \mathrm { c }}$ & $\mathrm{Li}^{7}, 33 \mathrm{~cm}$ \\
\hline NA24 & 1.33 & - & 0.19 & 0.45 & $300 \mathrm{GeV} / \mathrm{c}$ & $\mathrm{LH}_{2}, 1 \mathrm{~m}$ \\
\hline WA70 & 3.3 & - & 1.3 & 5.2 & $280 \mathrm{GeV} / \mathrm{c}$ & $\mathrm{LH}_{2}, 1 \mathrm{~m}$ \\
\hline LA6 & - & 1.0 & - & 1.6 & $315 \mathrm{GeV} / \mathrm{c}$ & $\mathrm{H}_{2}$ gas jet \\
\hline
\end{tabular}

for direct photon production. Table 2 shows the direct photon event sensitivity expected for Ei05 at the end of the present fixed target run. The E705 sensitivities are determined from the current data set and extrapolations based on it for running through Feb. 15, 1988. These sensitivities should allow E705 to probe direct photon production up to $p_{T} \approx 8 \mathrm{GeV} / \mathrm{c}$ for $\pi^{ \pm}$ and $p$. Also shown are the event sensitivities reported at this workshop by the CERN direct photon experiments in the same energy range as E705: NA24[12], WA70[13], and UA6[14]. Compared tc WA70, ETU5 should have 3-4 times better sensitivity for $\pi^{\ddagger}$ production and roughly the same sensitivity for $p$ production. UA6 has cleariy the best sensitivity for $\bar{p}$ production at these energies.

\section{Conclusion}

The goal of experiment E705 is to study the contribution of the annihilation and Compton processes to direct photon production at $\sqrt{s}=23.7 \mathrm{GeV}$ and to extract quark and gluon structure functions for the pion and nucleon tirrough the comparisor of $\pi^{-} / \pi^{+}$and $\bar{p} / p$ high $p_{T}$ photon production. E705 is presently collecting data and by the end of the data run should have the sensitivity to be able to make a significant statement about direct photon production for $p_{T}$ 's up to $8 \mathrm{GeV} / \mathrm{c}$. 


\section{References}

1' Fermilab Proposal E-705 (1981).

[2] S. Conetti, et al, these proceedings.

[3] J. F. Owens, Rev. Mod. Phys. 59, 465 (1987).

[4) T. Ferbel and W. R. Molson, Rev. Mod. Phys. EB, 181 (1984).

[5] R. Baier, these proceedings.

16] D. E. Wagoner, et al., Nucl. Inst. and Meth. A238 (1985) 315; B. Cox, et al., Nucl. Inst. and Meth. A238 (1585) 321; B. Cox, et al., Nucl. Inst. and Meth. 218 (1984) 487.

[7] C. M. Jenkins, et al., Proceedings of the Gas Sampling Calorimetry Workshop II, Fermilab, 201 (1985); R. Rameika, et al., Nucl. Inst. and Meth. A236 (1985) 42; B. Cox, et al., Nucl. Inst. and Meth. 219 (1984) 491.

[8] L. Fortney, Proceedings of the Calorimeter Calibration Workshop, Fermilab, 1 (1983).

[9] R. Rameika, Proceedings of the Workshop on Triggering, Data Acquisition and Computing for High Energy/High Luminosity Hadron-Hadron Colliders, Fermilab, 111 (1985).

[10] S. Conetti, M. Haire, and K. Kuchela, IEEE Trans. Nucl. Sci. NS-32 (1985) 1318;

S. Conetti and K. Kuchela, IEEE Trans. Nucl. Sci. NS-32 (1985) 1326.

[11] H. Areti, et al., Nucl. Inst. and Meth. 212 (1983) 135.

(12] P. Seyboth, these proceedings; C. De Marzo, et al., Phys. Rev. D 36, 8 (1987).

[13] M. Martin, these proceedings; M. Bonesini, et al., CERN-EP/87-185 (1987).

[14] P. T. Cox, these proceedings. 


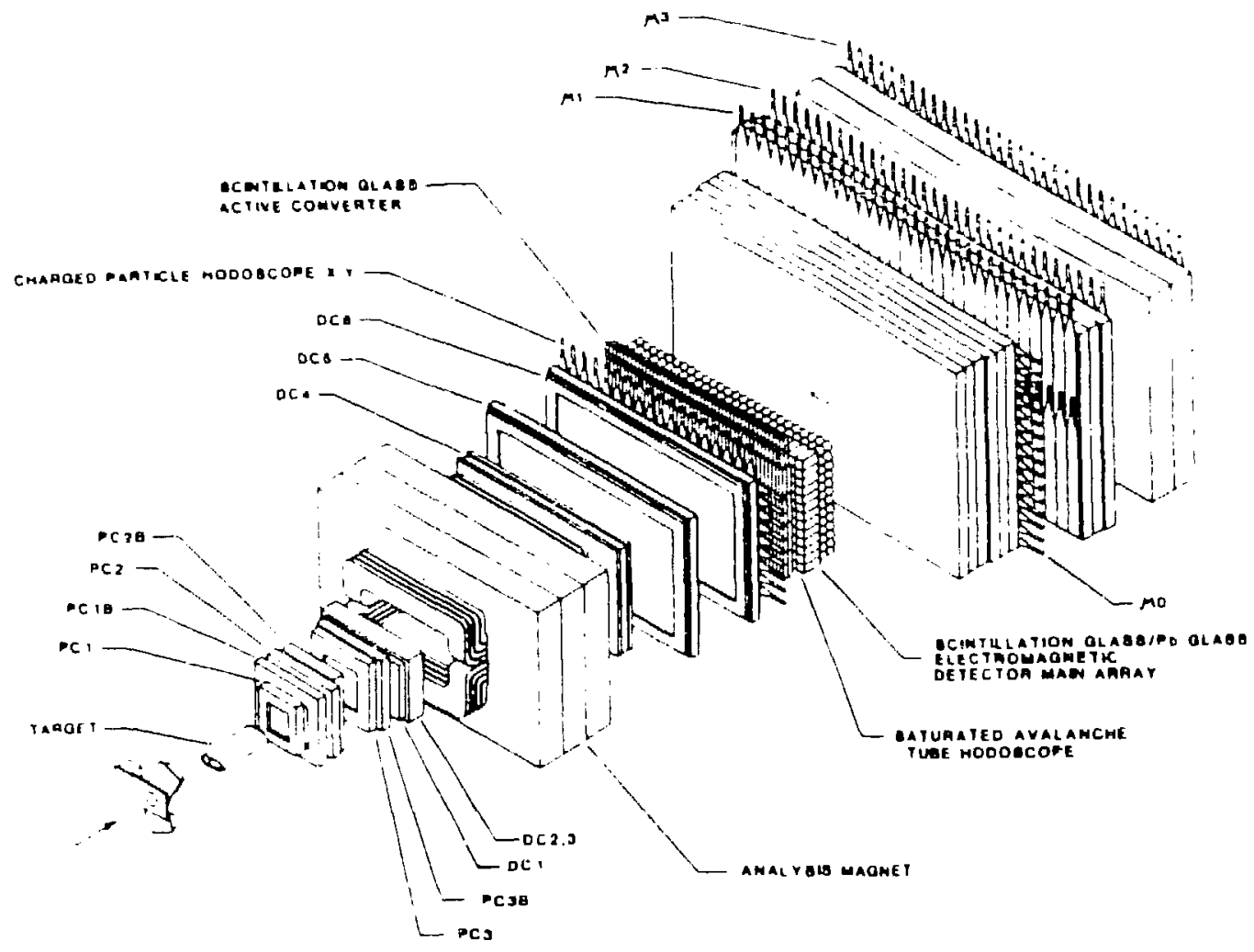

Figure 1: Experimental Layout of E705.

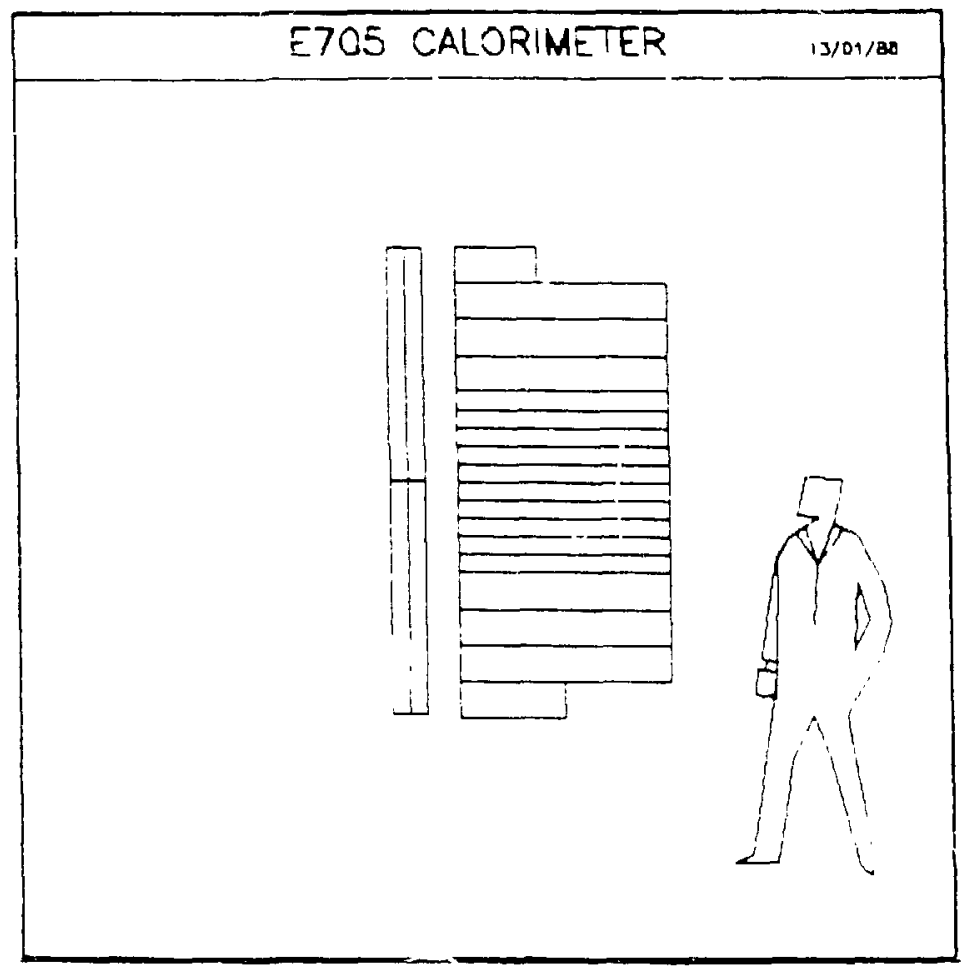

Figure 2: Side view of E705 electromagnetic calorimeter. The beam enters from the ieft. Shown are the two active converter layers and the main array. 
Figure 3: Front view of E705 Main Array.

a)

Cose $\left(10^{6}\right.$ rods $)$
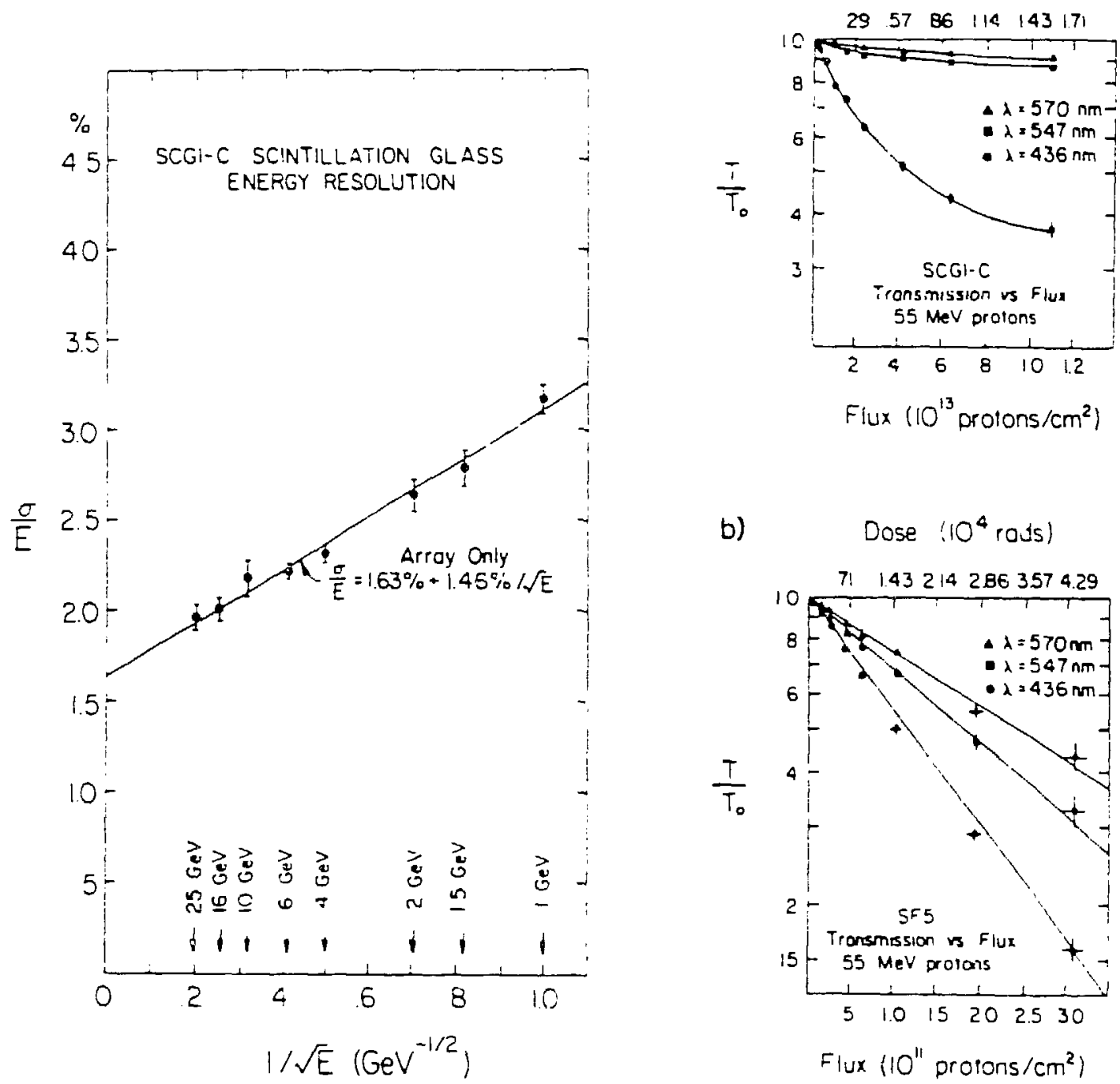

b) Dose $\left(10^{4}\right.$ rojs $)$

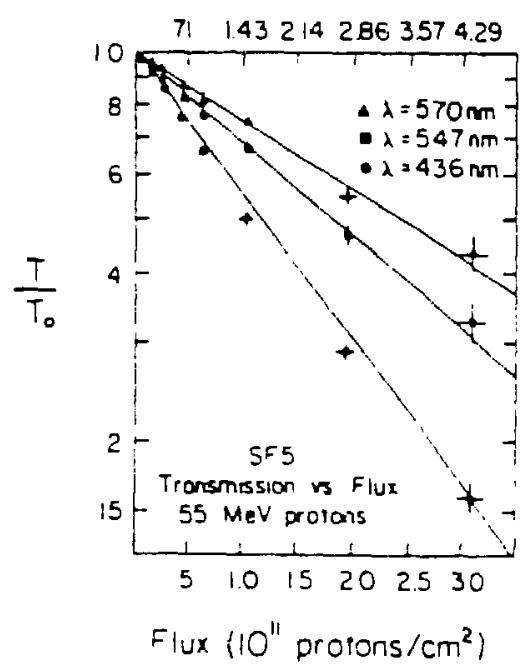

Figure 4: Energy Resolution for an

Figure 5: Optica! transmission along the beam axis at the specified waveleugth for $1 \mathrm{~cm}$ thick samples of (a) SCG1-C and (b) SF5 as a function of dose. 


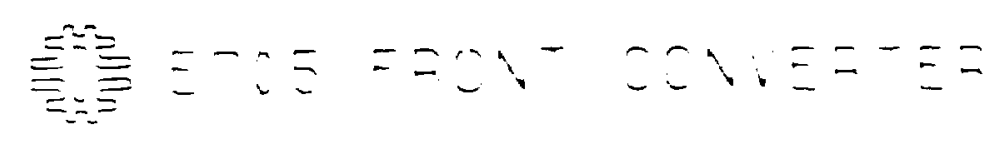

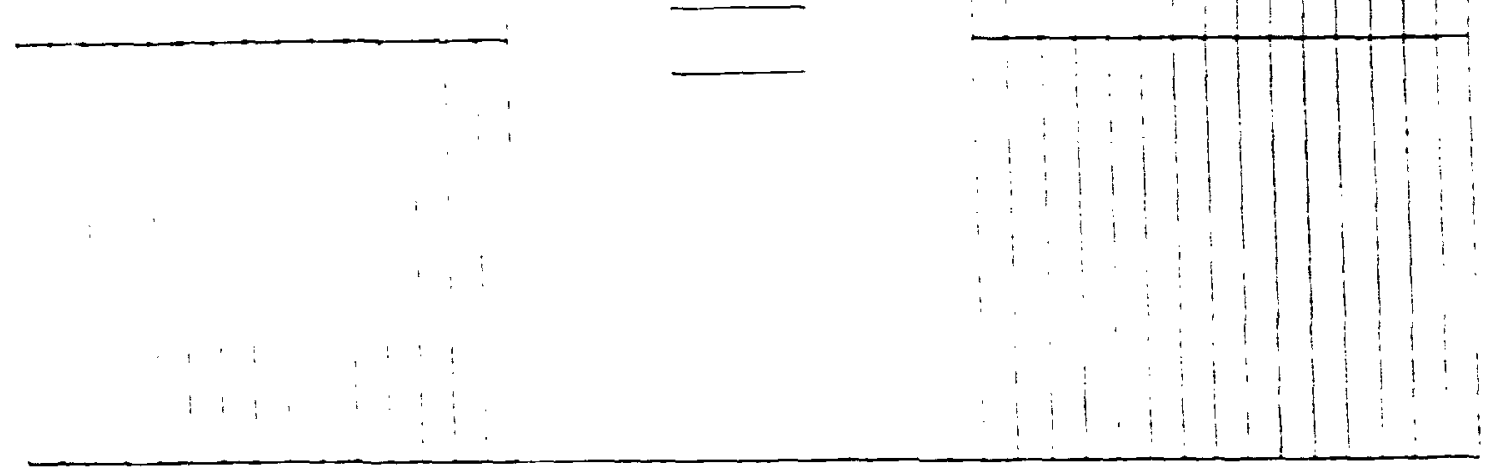

Figure 6: Front riew of E705 active converter and lead-gas converter.

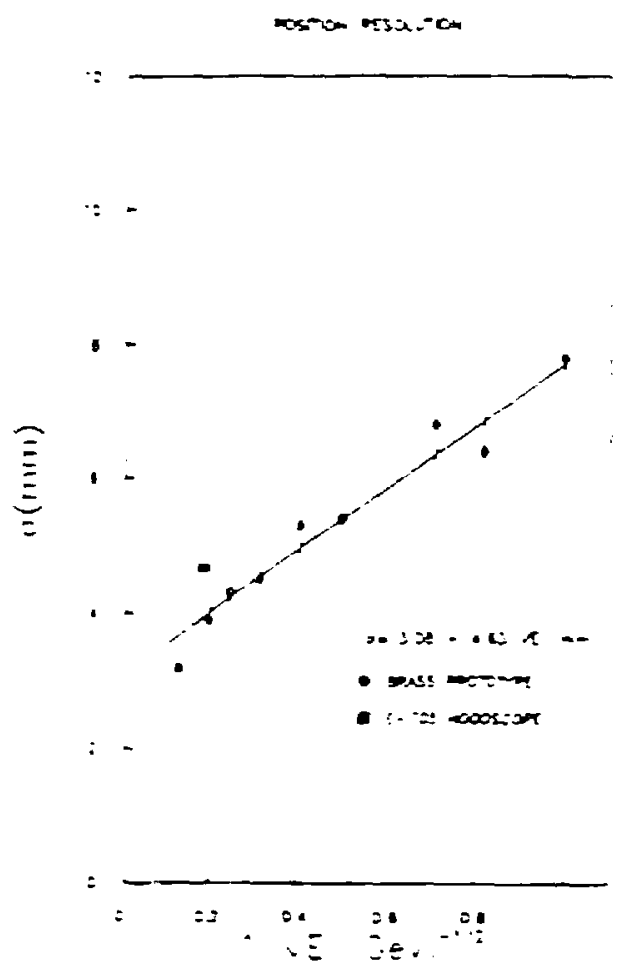

Figure 7: Position resolution ws energy for the E705 gas tude hodoscope. 


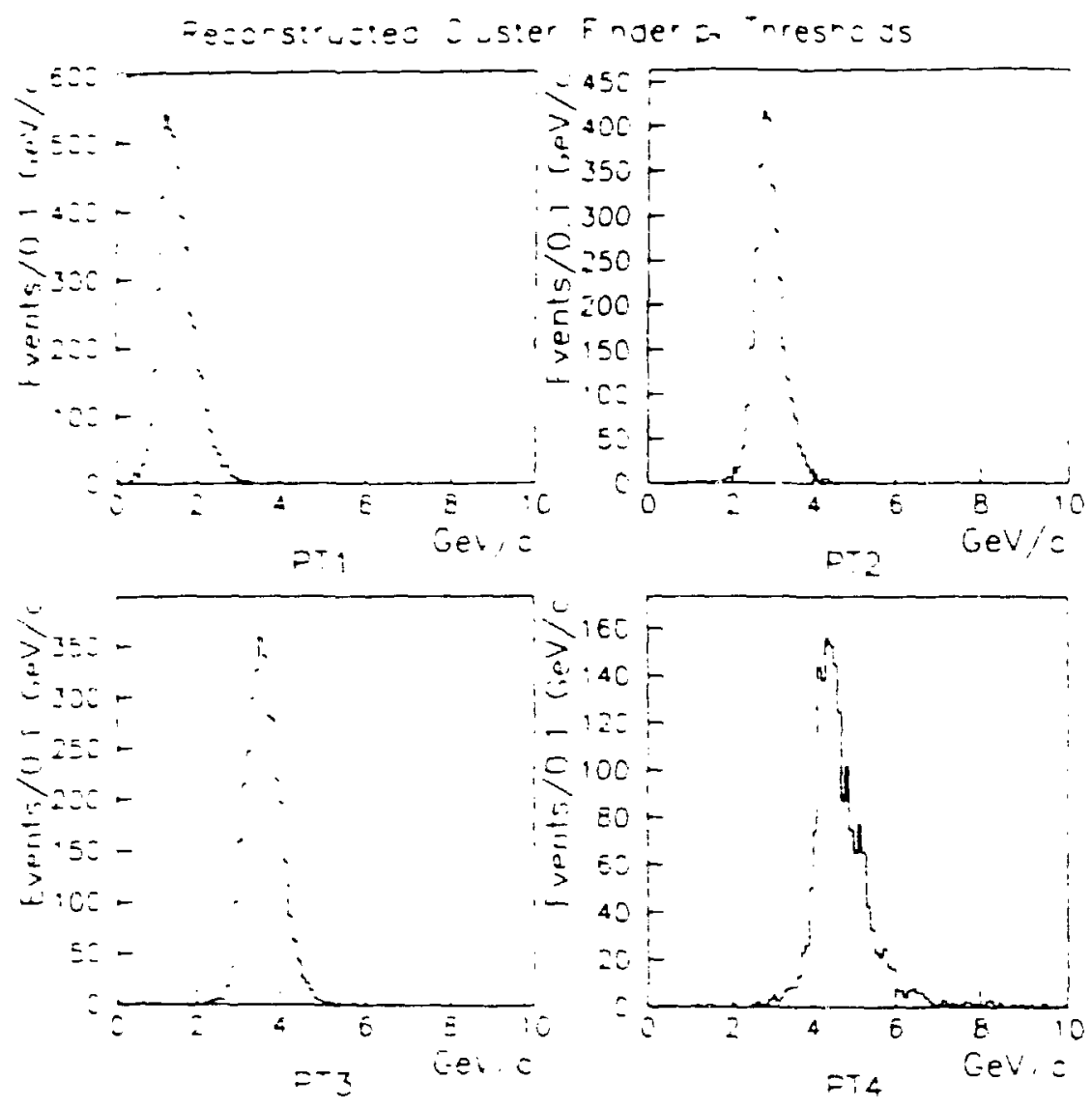

Figure 8: Raw uncorrected $p_{T}$ sums for $p_{T}$ trigger thresholds of: $\mathrm{PT} 1=1.7 \mathrm{GeV} / \mathrm{c}, \mathrm{PT} 2=2.5 \mathrm{GeV} / \mathrm{c}$. PT3 $=3.5 \mathrm{GeV} / \mathrm{c}$, $\mathrm{PT} 4=4.5 \mathrm{GeV}$ ic.

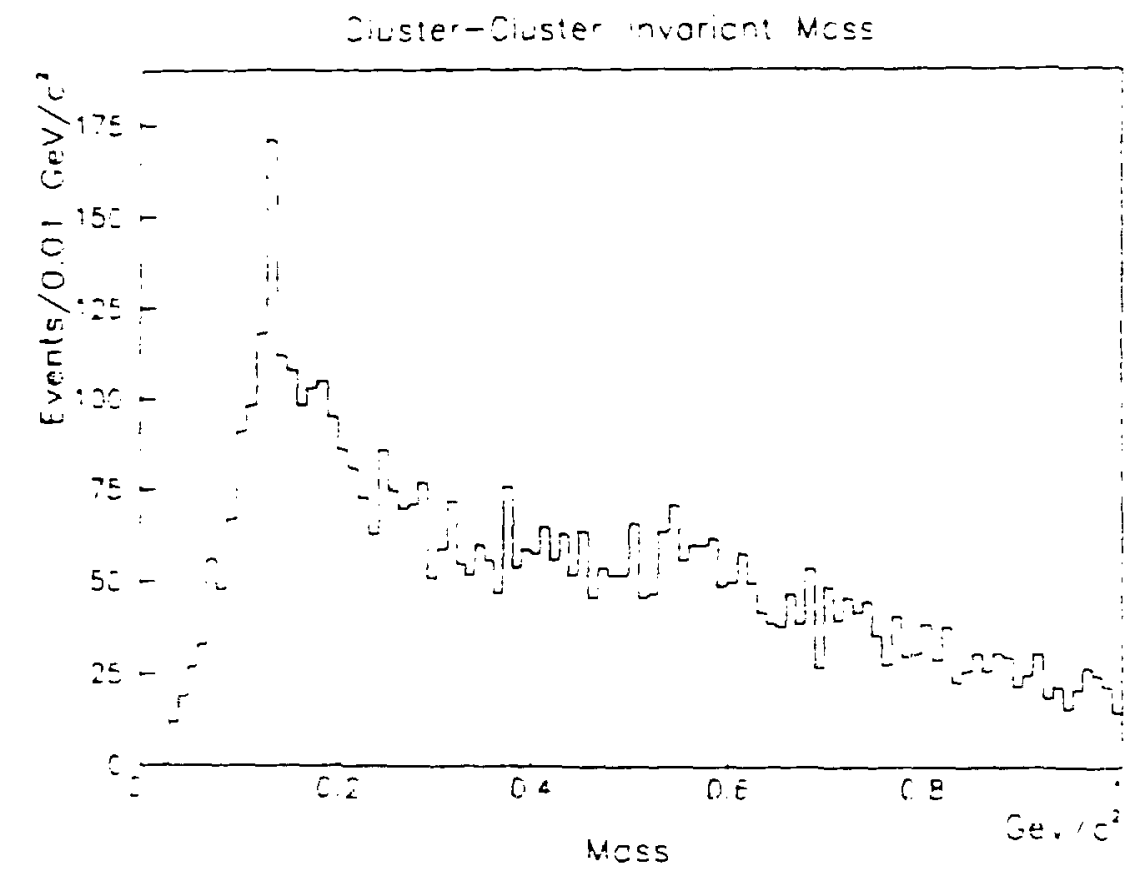

Figure 9: Preliminary invariant mass for all combinations of elusters outside of $=52.5 \mathrm{~cm}$ for PT1 and PT2 single photon triggers. No other cuts on the mass combinations are made. 